\title{
Research Article \\ Some new integral inequalities via general forms of proportional fractional integral operators
}

\author{
Saad Ihsan Butt ${ }^{1}$, Ahmet Ocak Akdemir ${ }^{2, *}$, Sinan Aslan ${ }^{3}$, Imdat İscan ${ }^{4}$, Praveen Agarwal ${ }^{5,6}$ \\ ${ }^{1}$ COMSATS University Islamabad, Lahore Campus, Pakistan \\ ${ }^{2}$ Department of Mathematics, Faculty of Arts and Sciences, A $\breve{g r t}$ İbrahim Çeçen University, A $\breve{g r l}$, Turkey \\ ${ }^{3}$ Institute of Graduate Studies, Ağrı İbrahim Çeçen University, Ağrı, Turkey \\ ${ }^{4}$ Department of Mathematics, Faculty of Science and Arts, Giresun University, Giresun, Turkey \\ ${ }^{5}$ International Center for Basic and Applied Sciences, Jaipur, India \\ ${ }^{6}$ Department of Mathematics, Anand International College of Engineering, Jaipur, India
}

(Received: 7 May 2021. Received in revised form: 3 July 2021. Accepted: 5 July 2021. Published online: 10 July 2021.)

(C) 2021 the authors. This is an open access article under the CC BY (International 4.0) license (www.creativecommons.org/licenses/by/4.0/).

\section{Abstract}

In this paper, some new integral inequalities for integrable geometrically convex mappings via the general forms of proportional fractional integral operators are proved. Basic definitions, various classical inequalities and generalized proportional fractional integral operators are used to prove the main findings.

Keywords: $A G$-convexity; generalized proportional fractional operators; Hölder inequality; general Cauchy inequality.

2020 Mathematics Subject Classification: 26A33, 26D10, 26D15, 33B20.

\section{Introduction and preliminaries}

Fractional analysis has brought a new dimension to many fields in mathematics and has become one of the most popular topics in recent years with its applications in several disciplines such as engineering, physics, modeling and control theory. Researchers have started to work intensively on fractional integral and derivative operators, and many new concepts and new applications have been included in the literature. The new features added by each new operator tries to prove their effectiveness in the real world problems solutions and the adventure continues in the search for the most effective operators. Many studies were conducted with the help of these operators to explain physical phenomena and demonstrate wide usage area in inequality theory (see the papers $[3,7,12,19,20,24,27,30,34-36])$. Now, we take a look at fractional integrals from a historical perspective by recalling these operators.

Definition 1.1. Let $f \in L_{1}[a, b]$. The Riemann-Liouville integrals $J_{a+}^{\alpha} f$ and $J_{b-}^{\alpha} f$ of order $\alpha>0$ with $a \geq 0$ are defined by

$$
J_{a+}^{\alpha} f(\epsilon)=\frac{1}{\Gamma(\alpha)} \int_{a}^{\epsilon}(\epsilon-\kappa)^{\alpha-1} f(\kappa) d \kappa, \quad \epsilon>a
$$

and

$$
J_{b-}^{\alpha} f(\epsilon)=\frac{1}{\Gamma(\alpha)} \int_{\epsilon}^{b}(\kappa-\epsilon)^{\alpha-1} f(\kappa) d \kappa, \quad \epsilon<b
$$

respectively. Here $\Gamma(\epsilon)$ is the Gamma function and its definition is

$$
\Gamma(\epsilon)=\int_{0}^{\infty} e^{-\epsilon} \epsilon^{\kappa-1} d \kappa .
$$

It is to be noted that $J_{a+}^{0} f(\epsilon)=J_{b-}^{0} f(\epsilon)=f(\epsilon)$ in the case of $\alpha=1$, the fractional integral reduces to the classical integral.

Riemann-Liouville integral operators are presented as a generalization of classical integrals. Then a more general version of this useful operator is given as follows.

Definition 1.2. [22] Let $(a, b)$ with $-\infty<a<b<\infty$ be a finite or infinite interval of the real line $\mathbb{R}$ and $\alpha$ a complex number with $\operatorname{Re}(\alpha)>0$. Also let $h$ be a strictly increasing function on $(a, b)$, having a continuous derivative $h^{\prime}$ on $(a, b)$. The generalized left and right sided Riemann-Liouville fractional integrals of a function $f$ with respect to another function $h$ on $[a, b]$ defined as

$$
{ }_{a+}^{h} I^{\alpha} f(\epsilon)=\frac{1}{\Gamma(\alpha)} \int_{a}^{\epsilon}(h(\epsilon)-h(\kappa))^{\alpha-1} f(\kappa) h^{\prime}(\kappa) d \kappa, \quad \epsilon>a
$$


and

$$
{ }_{b-}^{h} I^{\alpha} f(\epsilon)=\frac{1}{\Gamma(\alpha)} \int_{\epsilon}^{b}(h(\kappa)-h(\epsilon))^{\alpha-1} f(\kappa) h^{\prime}(\kappa) d \kappa, \quad \epsilon<b .
$$

In [15], Jarad et al. investigated the generalized proportional fractional integrals with a different kernel structure that satify several important properties as follows:

Definition 1.3. The left and right generalized proportional fractional integral operators are respectively defined by

$$
{ }_{a+} \mathcal{J}^{\alpha, \zeta} f(\epsilon)=\frac{1}{\zeta^{\alpha} \Gamma(\alpha)} \int_{a}^{\epsilon} e^{\left[\frac{\zeta-1}{\zeta}(\epsilon-\kappa)\right]}(\epsilon-\kappa)^{\alpha-1} f(\kappa) d \kappa, \quad \epsilon>a
$$

and

where $\zeta \in(0,1]$ and $\alpha \in \mathbb{C}$ and $\mathbb{R}(\alpha)>0$.

$$
{ }_{b-} \mathcal{J}^{\alpha, \zeta} f(\epsilon)=\frac{1}{\zeta^{\alpha} \Gamma(\alpha)} \int_{\epsilon}^{b} e^{\left[\frac{\zeta-1}{\zeta}(\kappa-\epsilon)\right]}(\kappa-\epsilon)^{\alpha-1} f(\kappa) d \kappa, \quad \epsilon<b
$$

We will continue with the Hadamard integral operators and the Katugampola integral operators, which are a general variant of the Riemann-Liouville integral operators as follows:

Definition 1.4. [21] Let $a, b$ be two non-negative real numbers satisfying the inequality $a<b$, and $\alpha, \rho$ be two positive real numbers and $f:[a, b] \rightarrow \mathbb{R}$ be an integrable function. The left and right Katugampola fractional integrals defined as

$$
{ }_{a+} \mathcal{I}^{\alpha} f(\epsilon)=\frac{1}{\Gamma(\alpha)} \int_{a}^{\epsilon}\left(\frac{\epsilon^{\rho}-\kappa^{\rho}}{\rho}\right)^{\alpha-1} \frac{f(\kappa) d \kappa}{\kappa^{1-\rho}}, \quad \epsilon>a
$$

and

$$
{ }_{b-} \mathcal{I}^{\alpha} f(\epsilon)=\frac{1}{\Gamma(\alpha)} \int_{\epsilon}^{b}\left(\frac{\kappa^{\rho}-\epsilon^{\rho}}{\rho}\right)^{\alpha-1} \frac{f(\kappa) d \kappa}{\kappa^{1-\rho}}, \quad \epsilon<b .
$$

The associated integral operator based on the Hadamard derivative operator is given as follows.

Definition 1.5. [22,29] Let $a, b$ be two reals with $0<a<b$ and $f(\kappa):[a, b] \rightarrow \mathbb{R}$ be an integrable function. The left and right Hadamard fractional integrals of order $\alpha>0$ are defined as

$$
{ }_{a+} \mathfrak{F}^{\alpha} f(\epsilon)=\frac{1}{\Gamma(\alpha)} \int_{a}^{\epsilon} \frac{f(\kappa)}{\kappa\left(\ln \frac{\epsilon}{\kappa}\right)^{1-\alpha}} d \kappa, \quad \epsilon>a
$$

and

$$
{ }_{b-} \mathfrak{F}^{\alpha} f(\epsilon)=\frac{1}{\Gamma(\alpha)} \int_{\epsilon}^{b} \frac{f(\kappa)}{\kappa\left(\ln \frac{\kappa}{\epsilon}\right)^{1-\alpha}} d \kappa, \quad \epsilon<b .
$$

Within the scope of fractional analysis studies, the search for the operator with the most effective and general kernels led the researchers to define the operator named generalized proportional Hadamard fractional integrals. This operator, which has a different structure, is given as follows.

Definition 1.6. [26] The left and right generalized proportional Hadamard fractional integrals of order $\alpha>0$ and proportionality index $\zeta \in(0,1]$ is defined by

$$
{ }_{a+} \mathcal{F}^{\alpha, \zeta} f(\epsilon)=\frac{1}{\zeta^{\alpha} \Gamma(\alpha)} \int_{a}^{\epsilon} \frac{e^{\left[\frac{\zeta-1}{\zeta}\left(\ln \frac{\epsilon}{\kappa}\right)\right]}}{\left(\ln \frac{\epsilon}{\kappa}\right)^{1-\alpha}} \frac{f(\kappa)}{\kappa} d \kappa, \quad \epsilon>a
$$

and

$$
{ }_{b-} \mathcal{F}^{\alpha, \zeta} f(\epsilon)=\frac{1}{\zeta^{\alpha} \Gamma(\alpha)} \int_{a}^{\epsilon} \frac{e^{\left[\frac{\zeta-1}{\zeta}\left(\ln \frac{\kappa}{\epsilon}\right)\right]}}{\left(\ln \frac{\kappa}{\epsilon}\right)^{1-\alpha}} \frac{f(\kappa)}{\kappa} d \kappa, \quad \epsilon<b .
$$

On all of these, the generalized proportional fractional (GPF) integral operator in the sense of another function $h$ has been defined as follows.

Definition 1.7. $[18,28]$ Let $f \in X_{h}^{q}(0, \infty)$, there is an increasing, positive monotone function $h$ defined on $[0, \infty)$ having continuous derivative $h^{\prime}$ on with $h(0)=0$. Then the left-sided and right-sided GPF-integral operator of a function $f$ in the sense of another function $h$ of order $\alpha>0$ are stated as:

$$
{ }_{a+\mathfrak{J}}^{h} \mathfrak{J}^{\alpha, \zeta} f(\epsilon)=\frac{1}{\zeta^{\alpha} \Gamma(\alpha)} \int_{a}^{\epsilon} e^{\left[\frac{\zeta-1}{\zeta}(h(\epsilon)-h(\kappa))\right]}(h(\epsilon)-h(\kappa))^{\alpha-1} f(\kappa) h^{\prime}(\kappa) d \kappa, \quad \epsilon>a
$$

and

$$
{ }_{b-}^{h} \mathfrak{J}^{\alpha, \zeta} f(\epsilon)=\frac{1}{\zeta^{\alpha} \Gamma(\alpha)} \int_{\epsilon}^{b} e^{\left[\frac{\zeta-1}{\zeta}(h(\kappa)-h(\epsilon))\right]}(h(\kappa)-h(\epsilon))^{\alpha-1} f(\kappa) h^{\prime}(\kappa) d \kappa, \quad \epsilon<b
$$

where $\zeta \in(0,1]$ and $\alpha \in \mathbb{C}$ and $\mathbb{R}(\alpha)>0$. 
If we set the parameters with different choices in Definition 1.7, one can obtain Riemann-Liouville integrals, generalized Riemann-Liouville fractional integrals, generalized proportional fractional integrals, Katugampola fractional integrals, Hadamard fractional integrals and generalized proportional Hadamard fractional integrals.

In [25], Pečarić et al. mentioned about some different classes of convex functions as followings:

A function $f: I \rightarrow[0, \infty)$ is said to be $\log$-convex or multiplicatively convex ( $A G$-convex) if $\log f$ is convex, or, equivalently, for all $x, y \in I$ and $t \in[0,1]$ one has the inequality:

$$
f(t x+(1-t) y) \leq f(x)^{t} f(y)^{1-t} .
$$

A function $f: I \rightarrow[0, \infty)$ is said to be $G A$-convex if for all $x, y \in I$ and $t \in[0,1]$, one has the inequality:

$$
f\left(x^{t} y^{1-t}\right) \leq t f(x)+(1-t) f(y) .
$$

Example 1.1. The function $f(x)=\frac{1}{x}, x \in(0,1)$ is log-convex on $(0,1)$.

The researchers have performed numerous research articles on various integral inequalities by using different kinds of fractional integral operators with applications, see [1,2,4-6, 8-11,13,14,16-18, 23, 28,31-33].

The main aim of this paper is to establish some new integral inequalities for product of two geometrically convex functions via the general forms of proportional fractional integral operators.

\section{Main results}

Theorem 2.1. Assume that $f, g:(0, \infty) \rightarrow R$ be differentiable function and $\psi$ be a positive monotone increasing function that defined on $[0, \infty)$. Let $\psi^{\prime}(\tau)$ be continuous and $\psi(0)=0, \quad p, q>1, \quad \frac{1}{p}+\frac{1}{q}=1,\left|\psi^{\prime}(\tau)\right| \leq M$. Then, if $f$ and $g$ are $A G-$ convex functions, we have the following inequality;

$$
\begin{aligned}
\left({ }^{\Psi} \mathcal{T}_{0^{+}, \tau}^{\eta, \xi} f g\right)(\tau) \leq & \frac{f g(b)-f g(a)}{b-a} \frac{M^{\frac{p-1}{p}}}{\xi^{\eta} \Gamma(\eta)}\left(\frac{e^{\frac{\xi-1}{\xi} p \psi(\tau)}-1}{\frac{\xi-1}{\xi} p}\right)^{\frac{1}{p}}\left(\int_{0}^{\tau}\left|(\psi(\tau)-\psi(x))^{\eta-1}(x-b)\right|^{q} d x\right)^{\frac{1}{q}} \\
& +\frac{f g(b)}{\xi^{\eta} \Gamma(\eta)} \frac{\psi^{\eta}(\tau)}{\left(\frac{1-\xi}{\xi} \psi(\tau)\right)^{\eta}}\left(\Gamma(\eta)-\Gamma\left(\eta, \frac{1-\xi}{\xi} \psi(\tau)\right)\right)
\end{aligned}
$$

for $\xi \in(0,1), \eta \in \mathbb{C}, \operatorname{Re}(\eta)>0, \tau>0$.

Proof. By using the definition of AG-convex functions, we can write

$$
f g(t a+(1-t) b) \leq[f g(a)]^{t}[f g(b)]^{1-t} .
$$

By changing of the variable such that $x=t a+(1-t) b$, we have

$$
f g(x) \leq[f g(a)]^{\frac{b-x}{b-a}}[f g(b)]^{\frac{x-a}{b-a}} .
$$

By applying the General Cauchy inequality to above inequality, we get

$$
f g(x) \leq\left(\frac{b-x}{b-a}\right) f g(a)+\left(\frac{x-a}{b-a}\right) f g(b) .
$$

Namely

$$
f g(x) \leq \frac{f g(b)-f g(a)}{b-a}(x-b)+f g(b) .
$$

If we denote $A=\frac{f g(b)-f g(a)}{b-a}$ and $B=f g(b)$, we get

$$
f g(x) \leq A(x-b)+B .
$$

By multiplying both sides of the resulting inequality with

$$
\frac{1}{\xi^{\eta} \Gamma(\eta)} \int_{0}^{\tau} \frac{e^{\frac{\xi-1}{\xi}(\psi(\tau)-\psi(x))}}{(\psi(\tau)-\psi(x))^{1-\eta}} \psi^{\prime}(x) d x,
$$

we get

$$
\left({ }^{\Psi} \mathcal{T}_{0^{+}, \tau}^{\eta, \xi} f g\right)(\tau) \leq \frac{A}{\xi^{\eta} \Gamma(\eta)} \int_{0}^{\tau} \frac{e^{\frac{\xi-1}{\xi}(\psi(\tau)-\psi(x))}}{(\psi(\tau)-\psi(x))^{1-\eta}}(x-b) \psi^{\prime}(x) d x+\frac{B}{\xi^{\eta} \Gamma(\eta)} \int_{0}^{\tau} \frac{e^{\frac{\xi-1}{\xi}(\psi(\tau)-\psi(x))}}{(\psi(\tau)-\psi(x))^{1-\eta}} \psi^{\prime}(x) d x
$$


By applying the well known Hölder inequality, we get

$$
\begin{aligned}
\left({ }^{\Psi} \mathcal{T}_{0^{+}, \tau}^{\eta, \xi} f g\right)(\tau) \leq & \frac{A}{\xi^{\eta} \Gamma(\eta)}\left(\int_{0}^{\tau}\left|e^{\frac{\xi-1}{\xi}(\psi(\tau)-\psi(x))} \psi^{\prime}(x)\right|^{p} d x\right)^{\frac{1}{p}}\left(\int_{0}^{\tau}\left|(\psi(\tau)-\psi(x))^{\eta-1}(x-b)\right|^{q} d x\right)^{\frac{1}{q}} \\
& +\frac{B}{\xi^{\eta} \Gamma(\eta)} \int_{0}^{\tau} \frac{e^{\frac{\xi-1}{\xi}(\psi(\tau)-\psi(x))}}{(\psi(\tau)-\psi(x))^{1-\eta}} \psi^{\prime}(x) d x .
\end{aligned}
$$

By making use of some necessary operation and by taking into account $\left|\psi^{\prime}(x)\right| \leq M$, we obtain

$$
\begin{aligned}
\left({ }^{\Psi} \mathcal{T}_{0^{+}, \tau}^{\eta, \xi} f g\right)(\tau) \leq & \frac{f g(b)-f g(a)}{b-a} \frac{M^{\frac{p-1}{p}}}{\xi^{\eta} \Gamma(\eta)}\left(\frac{e^{\frac{\xi-1}{\xi} p \psi(\tau)}-1}{\frac{\xi-1}{\xi} p}\right)^{\frac{1}{p}}\left(\int_{0}^{\tau}\left|(\psi(\tau)-\psi(x))^{\eta-1}(x-b)\right|^{q} d x\right)^{\frac{1}{q}} \\
& +\frac{f g(b)}{\xi^{\eta} \Gamma(\eta)} \frac{\psi^{\eta}(\tau)}{\left(\frac{1-\xi}{\xi} \psi(\tau)\right)^{\eta}}\left(\Gamma(\eta)-\Gamma\left(\eta, \frac{1-\xi}{\xi} \psi(\tau)\right)\right) .
\end{aligned}
$$

The proof is completed.

Theorem 2.2. Assume that $f, g:(0, \infty) \rightarrow R$ be differentiable function and $\psi$ be a positive monoton increasing function that defined on $[0, \infty)$. Let $\psi^{\prime}(\tau)$ be continuous and $\psi(0)=0, p, q>1, \frac{1}{p}+\frac{1}{q}=1,\left|\psi^{\prime}(\tau)\right| \leq M$. Then, if $f$ and $g$ are $A G-$ convex functions, we have the following inequality;

$$
\begin{aligned}
\left({ }^{\Psi} \mathcal{T}_{0^{+}, \tau}^{\eta, \xi} f g\right)(\tau) \leq & \left.\frac{f g(b)-f g(a)}{b-a} \frac{M^{\frac{p-1}{p}}}{\xi^{\eta} \Gamma(\eta)}\left(\frac{\psi^{p(\eta-1)+1}(\tau)}{\left(\frac{1-\xi}{\xi} p \psi(\tau)\right)^{p(\eta-1)+1}}\left(\Gamma(p(\eta-1)+1)-\Gamma\left(p(\eta-1)+1, \frac{1-\xi}{\xi} p \psi(\tau)\right)\right)\right)\right)^{\frac{1}{p}} \\
& \times\left(\frac{|\tau-b|^{q+1}-|-b|^{q+1}}{q+1}\right)^{\frac{1}{q}}+\frac{f g(b)}{\xi^{\eta} \Gamma(\eta)} \frac{\psi^{\eta}(\tau)}{\left(\frac{1-\xi}{\xi} \psi(\tau)\right)^{\eta}}\left(\Gamma(\eta)-\Gamma\left(\eta, \frac{1-\xi}{\xi} \psi(\tau)\right)\right)
\end{aligned}
$$

for $\xi \in(0,1), \eta \in \mathbb{C}, \operatorname{Re}(\eta)>0, \operatorname{Re}(p(\eta-1))>0, \tau>0$.

Proof. By using the definition of $A G$-convexity, we can write

$$
f g(t a+(1-t) b) \leq[f g(a)]^{t}[f g(b)]^{1-t} .
$$

By changing of the variable, we get

$$
f g(x) \leq[f g(a)]^{\frac{b-x}{b-a}}[f g(b)]^{\frac{x-a}{b-a}} .
$$

By applying General Cauchy inequality and by making some operations, we have

$$
f g(x) \leq \frac{f g(b)-f g(a)}{b-a}(x-b)+f g(b) .
$$

If we denote $A=\frac{f g(b)-f g(a)}{b-a}, B=f g(b)$, then

$$
f g(x) \leq A(x-b)+B .
$$

By multiplying both sides of the above inequality

$$
\frac{1}{\xi^{\eta} \Gamma(\eta)} \int_{0}^{\tau} \frac{e^{\frac{\xi-1}{\xi}(\psi(\tau)-\psi(x))}}{(\psi(\tau)-\psi(x))^{1-\eta}} \psi^{\prime}(x) d x
$$

we obtain

$$
\left({ }^{\Psi} \mathcal{T}_{0^{+}, \tau}^{\eta, \xi} f g\right)(\tau) \leq \frac{A}{\xi^{\eta} \Gamma(\eta)} \int_{0}^{\tau} \frac{e^{\frac{\xi-1}{\xi}(\psi(\tau)-\psi(x))}}{(\psi(\tau)-\psi(x))^{1-\eta}}(x-b) \psi^{\prime}(x) d x+\frac{B}{\xi^{\eta} \Gamma(\eta)} \int_{0}^{\tau} \frac{e^{\frac{\xi-1}{\xi}(\psi(\tau)-\psi(x))}}{(\psi(\tau)-\psi(x))^{1-\eta}} \psi^{\prime}(x) d x .
$$

By applying Hölder inequality to the resulting inequality, we get

$$
\left({ }^{\Psi} \mathcal{T}_{0^{+}, \tau}^{\eta, \xi} f g\right)(\tau) \leq \frac{A}{\xi^{\eta} \Gamma(\eta)}\left(\int_{0}^{\tau}\left|\frac{e^{\frac{\xi-1}{\xi}(\psi(\tau)-\psi(x))}}{(\psi(\tau)-\psi(x))^{1-\eta}} \psi(x)\right|^{p} d x\right)^{\frac{1}{p}}\left(\int_{0}^{\tau}|(x-b)|^{q} d x\right)^{\frac{1}{q}}
$$




$$
+\frac{B}{\xi^{\eta} \Gamma(\eta)} \int_{0}^{\tau} \frac{e^{\frac{\xi-1}{\xi}(\psi(\tau)-\psi(x))}}{(\psi(\tau)-\psi(x))^{1-\eta}} \psi^{\prime}(x) d x .
$$

By using the boundedness of $\left|\psi^{\prime}(x)\right| \leq M$ and some further calculation, we have

$$
\begin{aligned}
\left({ }^{\Psi} \mathcal{T}_{0^{+}, \tau}^{\eta, \xi} f g\right)(\tau) \leq & \frac{f g(b)-f g(a)}{b-a} \frac{M^{\frac{p-1}{p}}}{\xi^{\eta} \Gamma(\eta)}\left(\frac{\psi^{p(\eta-1)+1}(\tau)}{\left(\frac{1-\xi}{\xi} p \psi(\tau)\right)^{p(\eta-1)+1}}\left(\Gamma(p(\eta-1)+1)-\Gamma\left(p(\eta-1)+1, \frac{1-\xi}{\xi} p \psi(\tau)\right)\right)\right)^{\frac{1}{p}} \\
& \times\left(\frac{|\tau-b|^{q+1}-|-b|^{q+1}}{q+1}\right)^{\frac{1}{q}}+\frac{f g(b)}{\xi^{\eta} \Gamma(\eta)} \frac{\psi^{\eta}(\tau)}{\left(\frac{1-\xi}{\xi} \psi(\tau)\right)^{\eta}}\left(\Gamma(\eta)-\Gamma\left(\eta, \frac{1-\xi}{\xi} \psi(\tau)\right)\right) .
\end{aligned}
$$

This is the desired result.

Theorem 2.3. Assume that $f, g:(0, \infty) \rightarrow R$ be differentiable function and $\psi$ be a positive monoton increasing function that defined on $[0, \infty)$. Let $\psi^{\prime}(\tau)$ be continuous and $\psi(0)=0, \quad p, q>1$ ve $\frac{1}{p}+\frac{1}{q}=1,\left|\psi^{\prime}(\tau)\right| \leq M$. Then, if $f$ and $g$ are $G A-c o n v e x$ functions, we have the following inequality;

$$
\begin{aligned}
\left({ }^{\Psi} \mathcal{T}_{0^{+}, \tau}^{\eta, \xi} f g\right)(\tau) \leq & \frac{A}{\xi^{\eta} \Gamma(\eta)}\left(\frac{M^{\frac{p-1}{P}}}{p} \frac{\psi^{p(\eta-1)+1}(\tau)}{\left(\frac{1-\xi}{\xi} p \psi(\tau)\right)^{p(\eta-1)+1}}\right. \\
& \left.\left(\Gamma(p(\eta-1)+1)-\Gamma\left(p(\eta-1)+1, \frac{1-\xi}{\xi} p \psi(\tau)\right)\right)+\frac{1}{q} \int_{0}^{\tau}\left|\log _{\frac{a}{b}} \frac{x}{b}\right|^{q} d x\right) \\
& +\frac{B}{\xi^{\eta} \Gamma(\eta)} \frac{\psi^{\eta}(\tau)}{\left(\frac{1-\xi}{\xi} \psi(\tau)\right)^{\eta}}\left(\Gamma(\eta)-\Gamma\left(\eta, \frac{1-\xi}{\xi} \psi(\tau)\right)\right)
\end{aligned}
$$

for $\xi \in(0,1), \eta \in \mathbb{C}, \operatorname{Re}(\eta)>0, \operatorname{Re}(p(\eta-1))>0, \tau>0$.

Proof. By using the definition of $G A$-convexity, we can write

$$
f g\left(a^{t} b^{1-t}\right) \leq t f g(a)+(1-t) f g(b) .
$$

By making use of some arrangements, we have

$$
f g(x) \leq \log _{\frac{a}{b}} \frac{x}{b} f g(a)+\left(1-\log _{\frac{a}{b}} \frac{x}{b}\right) f g(b) .
$$

Namely,

$$
f g(x) \leq \log _{\frac{a}{b}} \frac{x}{b}(f g(a)-f g(b))+f g(b) .
$$

By denoting $A=f g(a)-f g(b), B=f g(b)$, we get

$$
f g(x) \leq A \log _{\frac{a}{b}} \frac{x}{b}+B .
$$

By multiplying the both sides of the above inequality by

$$
\frac{1}{\xi^{\eta} \Gamma(\eta)} \int_{0}^{\tau} \frac{e^{\frac{\xi-1}{\xi}(\psi(\tau)-\psi(x))}}{(\psi(\tau)-\psi(x))^{1-\eta}} \psi^{\prime}(x) d x
$$

it yields,

$$
\left({ }^{\Psi} \mathcal{T}_{0^{+}, \tau}^{\eta, \xi} f g\right)(\tau) \leq \frac{A}{\xi^{\eta} \Gamma(\eta)} \int_{0}^{\tau} \frac{e^{\frac{\xi-1}{\xi}(\psi(\tau)-\psi(x))}}{(\psi(\tau)-\psi(x))^{1-\eta}} \log _{\frac{a}{b}} \frac{x}{b} \psi^{\prime}(x) d x+\frac{B}{\xi^{\eta} \Gamma(\eta)} \int_{0}^{\tau} \frac{e^{\frac{\xi-1}{\xi}(\psi(\tau)-\psi(x))}}{(\psi(\tau)-\psi(x))^{1-\eta}} \psi^{\prime}(x) d x .
$$

By applying the Young inequality, we obtain

$$
\begin{aligned}
\left(\Psi_{0^{+}, \tau}^{\eta, \xi} f g\right)(\tau) \leq & \frac{A}{\xi^{\eta} \Gamma(\eta)}\left(\frac{1}{p} \int_{0}^{\tau}\left|\frac{e^{\frac{\xi-1}{\xi}(\psi(\tau)-\psi(x))}}{(\psi(\tau)-\psi(x))^{1-\eta}} \psi(x)\right|^{p} d x+\frac{1}{q} \int_{0}^{\tau}\left|\log _{\frac{a}{b}} \frac{x}{b}\right|^{q} d x\right) \\
& +\frac{B}{\xi^{\eta} \Gamma(\eta)} \int_{0}^{\tau} \frac{e^{\frac{\xi-1}{\xi}(\psi(\tau)-\psi(x))}}{(\psi(\tau)-\psi(x))^{1-\eta}} \psi^{\prime}(x) d x
\end{aligned}
$$


By making use of necessary operation and changing of the vairables, we get

$$
\begin{aligned}
\left({ }^{\Psi} \mathcal{T}_{0^{+}, \tau}^{\eta, \xi} f g\right)(\tau) \leq & \frac{A}{\xi^{\eta} \Gamma(\eta)}\left(\frac{M^{\frac{p-1}{P}}}{p} \frac{\psi^{p(\eta-1)+1}(\tau)}{\left(\frac{1-\xi}{\xi} p \psi(\tau)\right)^{p(\eta-1)+1}}\right. \\
& \left.\left(\Gamma(p(\eta-1)+1)-\Gamma\left(p(\eta-1)+1, \frac{1-\xi}{\xi} p \psi(\tau)\right)\right)+\frac{1}{q} \int_{0}^{\tau}\left|\log _{\frac{a}{b}} \frac{x}{b}\right|^{q} d x\right) \\
& +\frac{B}{\xi^{\eta} \Gamma(\eta)} \frac{\psi^{\eta}(\tau)}{\left(\frac{1-\xi}{\xi} \psi(\tau)\right)^{\eta}}\left(\Gamma(\eta)-\Gamma\left(\eta, \frac{1-\xi}{\xi} \psi(\tau)\right)\right),
\end{aligned}
$$

which completes the proof.

\section{References}

[1] A. O. Akdemir, S. I. Butt, M. Nadeem, M. A. Ragusa, New general variants of Chebyshev type inequalities via generalized integral operators, Mathematics 9 (2021) Art\# 1220.

[2] A. O. Akdemir, A. Ekinci, E. Set, Conformable fractional integrals and related new integral inequalities, J. Nonlinear Convex Anal. 18 (2017) $661-674$.

[3] S. Belarbi, Z. Dahmani. On some new fractional integral inequalities, J. Inequal. Pure Appl. Math. 10 (2009) 1-12.

[4] S. I. Butt, A. O. Akdemir, J. Nasir, F. Jarad, Some Hermite-Jensen-Mercer like inequalities for convex functions through a certain generalized fractional integrals and related reults, Miskolc Math. Notes 21 (2020) 689-715.

[5] S. I. Butt, M. Nadeem, S. Qaisar, A. O. Akdemir, T. Abdeljawad, Hermite Jensen Mercer type inequalities for conformable integrals and related results, Adv. Differ. Equ. 2020 (2020) Art\# 501.

[6] S. I. Butt, M. Umar, S. Rashid, A. O. Akdemir, Y. M. Chu, New Hermite Jensen Mercer-type inequalities via $k$-fractional integrals, Adv. Differ. Equ. 2020 (2020) Art\# 635.

[7] Z. Dahmani, O. Mechouar, S. Brahami, Certain inequalities related to the Chebyshev's functional involving a Riemann-Liouville operator, Bull. Math. Anal. Appl. 3 (2011) 38-44.

[8] M. A. Dokuyucu, A fractional order alcoholism model via Caputo-Fabrizio derivative, AIMS Math. 5 (2020) $781-797$.

[9] M. A. Dokuyucu, D. Baleanu, E. Celik, Analysis of Keller-Segel model with Atangana-Baleanu fractional derivative, Filomat 32 (2018) $5633-5643$.

[10] M. A. Dokuyucu, E. Celik, H. Bulut, H. M. Baskonus, Cancer treatment model with the Caputo-Fabrizio fractional derivative, Eur. Phys. J. Plus 133 (2018) Art\# 92.

[11] M. A. Dokuyucu, H. Dutta, A fractional order model for Ebola Virus with the new Caputo fractional derivative without singular kernel, Chaos Solitons Fractals 134 (2020) Art\# 109717.

[12] S. S. Dragomir, Some integral inequalities of Gruss type, Indian J. Pure Appl. Math. 31 (2000) 397-415.

[13] A. Ekinci, M. E. Ozdemir, Some new integral inequalities via Riemann-Liouville integral operators, Appl. Comput. Math. 18 (2019) $288-295$.

[14] F. Jarad, T. Abdeljawad, Generalized fractional derivatives and Laplace transform, Discrete Contin. Dyn. Syst. Ser. S 13 (2020) 709-722.

[15] F. Jarad, T. Abdeljawad, J. Alzabut, Generalized fractional derivatives generated by a class of local proportional derivatives, Eur. Phys. J. Spec. Top. 226 (2017) 3457-3471.

[16] F. Jarad, T. Abdeljawad, D. Baleanu, Caputo-type modification of the Hadamard fractional derivatives, Adv. Differ. Equ. 2012 (2012) Art\# 142.

[17] F. Jarad, T. Abdeljawad, D. Baleanu, On the generalized fractional derivatives and their Caputo modification, J. Nonlinear Sci. Appl. 10 (2017) $2607-2619$.

[18] F. Jarad, M. A. Alqudah, T. Abdeljawad, On more general forms of proportional fractional operators, Open Math. 18 (2020) $167-176$.

[19] E. Kaçar, Z. Kaçar, H. Yıldırım, Integral inequalities for Riemann-Liouville fractional integrals of a function with respect to another function, Iran. J. Math. Sci. Inform. 13 (2018) 1-13.

[20] U. N. Katugampola, Approach to a generalized fractional integral, Appl. Math. Comput. 218 (2011) 860-865.

[21] U. N. Katugampola, A new approach to a generalized fractional derivatives, Bull. Math. Anal. Appl. 6 (2014) 1-15.

[22] A. A. Kilbas, H. M. Srivastava, J. J. Trujillo, Theory and Applications of Fractional Differential Equations, North-Holland Mathematics Studies, New York, 2006.

[23] D. Nie, S. Rashid, A. O. Akdemir, D. Baleanu, J. B. Liu, On some new weighted inequalities for differentiable exponentially convex and exponentially quasi-convex functions with applications, Mathematics 7 (2019) Art\# 727.

[24] S. K. Ntouyas, S. D. Purohit, J. Tariboon, Certain Chebyshev type integral inequalities involving Hadamard's fractional operators, Abstr. Appl. Anal. 2014 (2014) Art\# 249091.

[25] J. Pečarić, F. Proschan, Y. L. Tong, Convex Functions, Partial Orderings and Statistical Applications, Academic Press, Boston, 1992.

[26] G. Rahman, T. Abdeljawad, F. Jarad, A. Khan, K. S. Nisar, Certain inequalities via generalized proportional Hadamard fractional integral operators, Adv. Differ. Equ. 2019 (2019) Art\# 454.

[27] G. Rahman, Z. Ullah, A. Khan, E. Set, K. S. Nisar, Certain Chebyshev-type inequalities involving fractional conformable integral operators, Mathematics 7 (2019) Art\# 364.

[28] S. Rashid, F. Jarad, M. A. Noor, H. Kalsoom, Y. M. Chu, Inequalities by means of generalized proportional fractional integral operators with respect to another function, Mathematics 7 (2019) Art\# 1225.

[29] S. G. Samko, A. A. Kilbas, Normal densities, Linear Algebra Appl. 396 (2005) 317-328.

[30] E. Set, A. O. Akdemir, I. Mumcu, Chebyshev type inequalities for conformable fractional integrals, Miskolc Math. Notes 20 (2019) $1227-1236$.

[31] E. Set, A. O. Akdemir, F. Özata, Grüss type inequalities for fractional integral operator involving the extended generalized Mittag Leffler function, Appl. Comput. Math. 19 (2020) 402-414.

[32] E. Set, A. O. Akdemir, M. E. Özdemir, Simpson type integral inequalities for convex functions via Riemann-Liouville integrals, Filomat 31 (2017) 4415-4420.

[33] E. Set, S. I. Butt, A. O. Akdemir, A. Karaolan, T. Abdeljawad, New integral inequalities for differentiable convex functions via Atangana-Baleanu fractional integral operators, Chaos Solitons Fractals 143 (2021) Art\# 110554.

[34] E. Set, U. Katugampola, I. Mumcu, M. E. Özdemir, Best fractional generalization of some inequalitites related to the Chebyshev's functional, Preprint, Available at https: //www.researchgate.net/publication/331284225.

[35] E. Set, M. Z. Sarikaya, F. Ahmad, A generalization of Chebychev type inequalities for first differentiable mappings, Miskolc Math. Notes 12 (2011) 245-253.

[36] H. Yıldırım, Z. Kırtay, Ostrowski inequality for generalized fractional integral and related inequalities, Malatya J. Mat. 2 (2014) 322-329. 